\title{
Do fiscal variables affect fiscal expectations? Experiments with real world and lab data ${ }^{\text {is }}$
}

\author{
Michele Bernasconi ${ }^{\mathrm{c}}$, Oliver Kirchkamp ${ }^{\mathrm{a}, *}$, Paolo Paruolo ${ }^{\mathrm{b}}$ \\ a Friedrich-Schiller-University Jena, School of Economics and Business Administration, Carl-Zeiss-Str. 3, 07743 Jena, Germany \\ ${ }^{b}$ Department of Economics, University of Insubria, Via Ravasi 2, I-21100 Varese, Italy \\ c Dipartimento di Scienze Economiche, Università “Cà Foscari” Venezia, Cannaregio 873, I-30121 Venezia, Italy
}

\section{A R T I C L E I N F O}

\section{Article history:}

Received 5 September 2005

Received in revised form 7 February 2008

Accepted 10 November 2008

Available online 25 November 2008

\section{JEL classification:}

C91

D89

E62

H31

Keywords:

Experiments

Fiscal policy

Expectations

Panel data

\begin{abstract}
A B S T R A C T
We generate observable expectations about fiscal variables through laboratory experiments using real world data from several European countries as stimuli. We compare a VAR model of expectations for data that is presented in a fiscal frame with one for neutrally presented data. We test the validity of the setup and find that participants understand the meaning of the fiscal variables, but also that their ability to perceive the correct characteristics of fiscal policy is limited. Expectations are consistent neither with rational nor with purely adaptive expectations, but instead follow an augmented-adaptive scheme.
\end{abstract}

(c) 2008 Elsevier B.V. All rights reserved.

\section{Introduction}

Expectations on fiscal variables are crucial to understand the effect of fiscal policy on the private sector. Little, however, is known about the way people actually form expectations on fiscal variables. While many models are based on the hypothesis of rational expectations, expectations themselves are not easily observable. As a result, empirical tests of the rational expectations hypothesis are often indirect, involving tests of predicted relations between observables, such as relationships between fiscal variables and components of output.

A problem in this approach is that factors that are not part of the model may affect expectations and choices in real economies. This makes the identification of the effects of expectations difficult because "economists cannot observe all the data that economic agents do" (Seater, 1993, p. 164).

\footnotetext{
is Financial support from Deutsche Forschungsgemeinschaft through Sonderforschungs bereich 504 and partial financial support from Italian MIUR grants ex $60 \%$ is gratefully acknowledged. We thank the associate editor and two anonymous referees for valuable comments and insights which helped us when we prepared the revision of the paper.

* Corresponding author. Tel.: +49 3641 943241; fax: +49 3641943242.

E-mail addresses: bernasconi@unive.it (M. Bernasconi), oliver@kirchkamp.de (O. Kirchkamp), paolo.paruolo@uninsubria.it (P. Paruolo).
} 
This limitation is also relevant for expectation measures derived from opinion surveys. ${ }^{1}$ Moreover surveys suffer from lack of economic incentives to reveal true opinions, so that for various reasons respondents "may express judgments that are different from the ones they choose to act upon" (Pesaran, 1987, p. 209).

An alternative is to measure expectations in a controlled experiment. Expectations experiments have been conducted since as early as the sixties in a wide range of economic contexts. ${ }^{2}$ Many of these experiments ask participants to form expectations for artificial time series that are based on an abstract model. The advantage of using artificial time series is that the data generating process is known to the experimenter and can immediately be used as a benchmark. It is possible to find out in a straightforward way under which conditions participants form which kinds of expectations for a given abstract model. This convenience comes at a cost: within this framework one can study expectations only for the given abstract model. First, one has to assume that participants in an experiment understand the formal description of the abstract model, and second, one has to assume that decision makers use similar procedures when they form expectations for different, and not perfectly known, processes in the real world. To bridge the gap between abstract games in the laboratory and economic reality, experimenters can calibrate a specific model with parameters from the real world. Experiments on tax compliance (Alm and McKee, 2004) or on decisions over monetary policy (Lombardelli et al., 2002; Blinder and Morgan, 2005) follow this approach.

In this paper we present a laboratory experiment that is not based on an abstract model with parameters from the real world but on real time series. Compared with experiments where participants forecast abstract time series, the use of real world time series has advantages and disadvantages. An advantage is that we can observe expectations in an economic context with real economic data. If the process of expectation formation depends on the economic context or on the type of the data, then the expectations we can measure are closer to the ones that are relevant for real economic decisions. A disadvantage is that with real world time series, it is no longer obvious what rational expectations are. We no longer have the clean benchmark abstract time series would provide. Instead, we have to construct a benchmark with the help of an econometric model. To do that, we follow a standard approach and assume that the DGP can be described as a VAR. The estimated VAR of the field data will constitute our benchmark solution.

We use annual time series on taxes and public expenditures from 15 OECD countries. Given the novelty of the approach we carefully check the internal validity of the experiment. We also study the impact of learning, fatigue, gender, and field of study of the participants.

To understand and to structure the expectation formation process better we assume that the joint DGP of stimuli and expectations data can be described as a VAR. We find that participants fail to perceive the fiscal properties from the field, thus violating the 'rational expectation hypothesis'. Expectations are rather in line with 'augmented-adaptive models', introduced in the early eighties by various authors (see Pesaran).

The paper is organised as follows. Section 2 describes the setup of the experiment. Section 3 provides descriptive statistics of the results and discusses internal validity. Section 4.1 develops the econometric approach to analyse both the stimulus and the expectations data. Inference results are presented in Section 4.2. The last section summarises the external significance of the approach and of the findings.

\section{Experimental setup}

In the experiment participants are exposed to graphic representations of time series of annual fiscal variables taken from various European countries. Time is denoted $t$, stimuli are gross total taxes $T_{t}$, total public expenditure $G_{t}$ (including interest payments), public debt $B_{t}$, and change in the debt level $\Delta B_{t}=B_{t}-B_{t-1}$, all expressed as yearly percentage of GDP. We focus on the relationship between taxes and expenditure, denoted by the vector $x_{t}:=\left(T_{t}, G_{t}\right)^{\prime}$. Here and in the following $\Delta$ is the time difference.

Participants know that they are facing European countries, but they do not know which specific country and which period the series refer to. Participants are paid in each period according to the precision of their expectations. The better their expectations, the higher is their income. The payment scheme can be motivated as a utility that a hypothetical agent derives from consumption over the current and the previous period if this agent uses the expectations of our participant and maximises the utility function:

$$
u_{t}=\prod_{i=t-1}^{t}\left(\frac{3}{4} C_{i}+\frac{1}{4} G_{i}\right)
$$

\footnotetext{
${ }^{1}$ For example, surveys conducted by Grun (1991) and Allers et al. (1998) found widespread evidence of misinformation on the conduct of government fiscal policy.

2 Such experiments include expectations for prices (Fisher, 1962; Schmalensee, 1976), expectations about artificially constructed time series (Hey, 1994), expectations in normal-form games (e.g. Costa-Gomes and Crawford, 2001), about the provision of public-goods (Offerman et al., 1996; Croson, 2000), expectations in the cobweb economy (Hommes et al., 2007), on monetary policies (e.g. Marimon and Sunder, 1993; Bernasconi and Kirchkamp, 2000) or even fiscal policy (Swenson, 1997).
} 

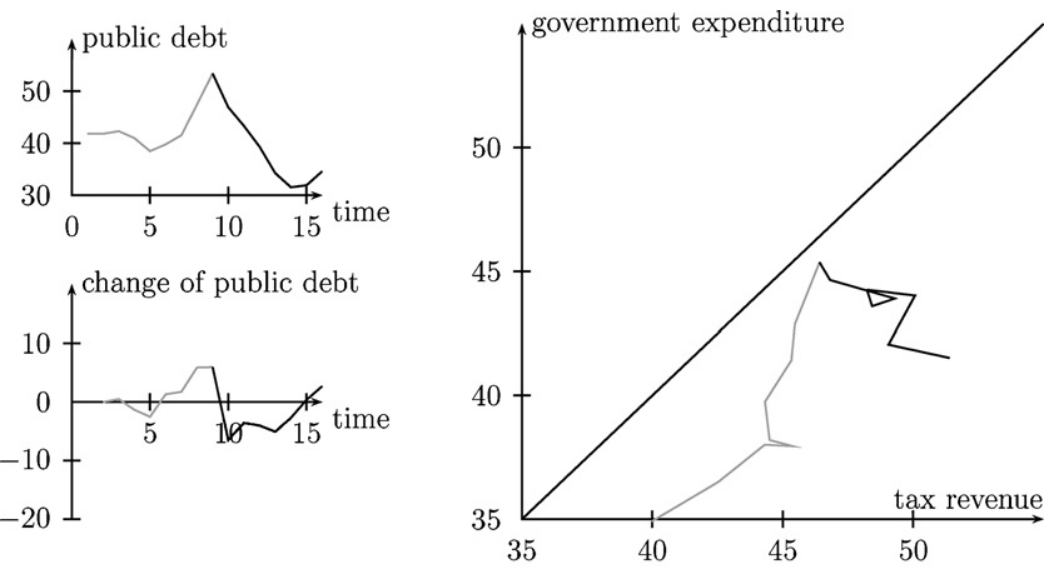

Values are given as percentage of GDP. Gray lines indicate past, black lines indicate recent years.

Fig. 1. $T_{t}$ and $G_{t}$ treatment. Values are given as percentage of GDP. Gray lines indicate past, black lines indicate recent years.

subject to the budget constraint:

$$
\sum_{i=t-1}^{t} \underbrace{\left(1-C_{i}-T_{i}\right)}_{\text {savings }} \cdot(1+r)^{i}=0 \quad \text { with } \quad r=0.1
$$

(where 1 is the normalised total GDP). Agents receive initial information on the first seven values of stimuli, which, for most countries, was the period from 1970 to 1976 . Let $t-1$ be the last available year and $X_{t-1}:=\left(x_{1}, \ldots, x_{t-1}\right)^{\prime}$ the available information; then forecasts are made repeatedly for each subsequent year $t$ until the end of the time series is reached in 1998. Participants would then start with another, randomly selected country.

We compare three experimental treatments. In a baseline treatment participants forecast both $T_{t}$ and $G_{t}$ (see Fig. 1). Forecasts for the next period are made by clicking with the mouse directly into the diagrams.

To control whether participants understand the economic context of the baseline treatment we also run a treatment where this context is removed. The time series are the same as in the baseline treatment, but variables have no economic significance. They are simply called $A, B$, and $C$. We call this treatment the 'neutral' treatment.

A main aspect of this paper is to study how participants use information about one variable (e.g. $T_{t}$ ) to forecast another (e.g. $G_{t}$ ). A natural implementation would be to present two variables $T_{t}$ and $G_{t}$ and to ask participants to forecast both. This is what we do in the above two treatments. To check whether such a simultaneous forecast is too difficult, we also run a control treatment where participants forecast $T_{t}$ only. ${ }^{3}$

Forecasts are called $T_{t}^{E_{i}}$ and $G_{t}^{E_{i}}$, where $i$ indicates agent $i$ and $E$ stands for expectation. The forecasts of agent $i$ that refer to time $t$ are denoted $y_{i, t}=\left(T_{t}^{E_{i}}, G_{t}^{E_{i}}\right)^{\prime}$ in the $T_{t}$ and $G_{t}$ treatment and $y_{i, t}:=T_{t}^{E_{i}}$ in the $T_{t}$ only treatment.

The time series of the stimuli (see Fig. 1) are updated every period after forecasts are made, so subjects learn about realizations of the stimuli as the economy moves on. Participants also get feedback about their wage, which reflects the accuracy of their forecast. ${ }^{4}$

Openly providing all payoff relevant information in economic experiments is standard and essential. Hence, we explain in the instructions (see Appendix) the above payment scheme in details with examples and also with a short technical note. Subjects were not obliged to look at the technical note, but were told that they could consult it and check everything if they wished to control the experimenter.

Stimulus data were from 15 European countries: Austria, Belgium, Denmark, Finland, France, Germany, Greece, Ireland, Italy, Netherlands, Norway, Portugal, Spain, Sweden, and UK. ${ }^{5}$ For the majority of countries the sample period of stimulus data was 1970-1998; a few exceptions (Denmark, France, Greece, Ireland, and UK) are due to limits in the availability of the

\footnotetext{
${ }^{3}$ In all three treatments subjects had information for past taxes and public expenditure. The display for the $T_{t}$ only treatment was similar to that of the $T_{t}$ and $G_{t}$ treatment in Fig. 1, with the only difference that the values of taxes and public expenditure were both displayed on the $y$-axis, with the period of the observations (not the year, which remained unknown to subjects) on the $x$-axis.

${ }^{4}$ To reward participants, the computer constructs the optimal consumption level $C_{t-1}$ based on a subjects' forecasts $y_{i, t}$ for year $t$ and given Eqs. (1) and (2). When in the next period $x_{t}:=\left(T_{t}, G_{t}\right)^{\prime}$ becomes available, we use Eqs. (2) and (1) to calculate $C_{t}$ and the utility $u_{t}$. The participant's per minute wage is $w=0.66 \times\left(u_{t} / u_{t}^{*}\right)^{\eta}$ where $\eta=12,000$ in the $T_{t}$ and $G_{t}$ treatment and 15,000 in the $T_{t}$ only treatment. $u_{t}^{*}$ is the utility the participant would obtain with forecasting the true values. This transformation from utilities into wages is monotonic and, hence, does not affect the maximisation problem of the individual, but creates steeper incentives to make good forecasts. Participants are paid this wage up to 2 min for each forecast. Parameters of the payment scheme have been developed in pilot studies previous to the experiment.

5 All stimulus data used in the experiment were taken from the database "Fiscal Positions and Business Cycles" (OECD, 2000).
} 

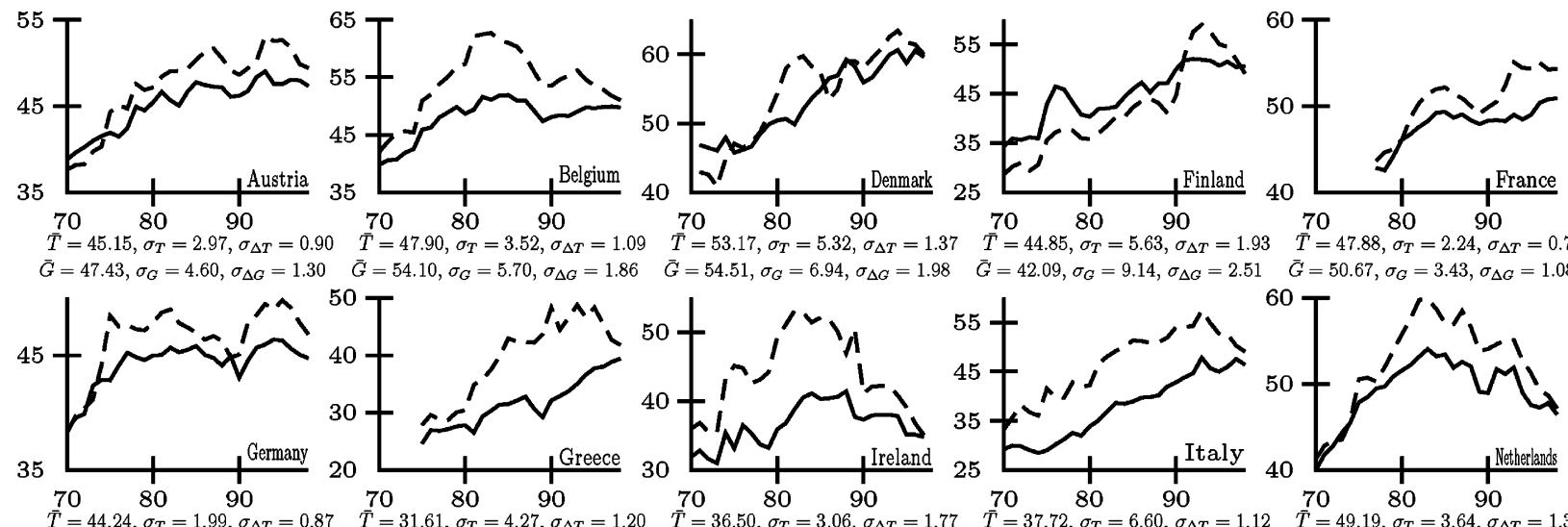

$\bar{G}=42.09, \sigma_{G}=9.14, \sigma_{\Delta G}=2.51 \quad \bar{G}=50.67, \sigma_{G}=3.43, \sigma_{\Delta G}=1.08$
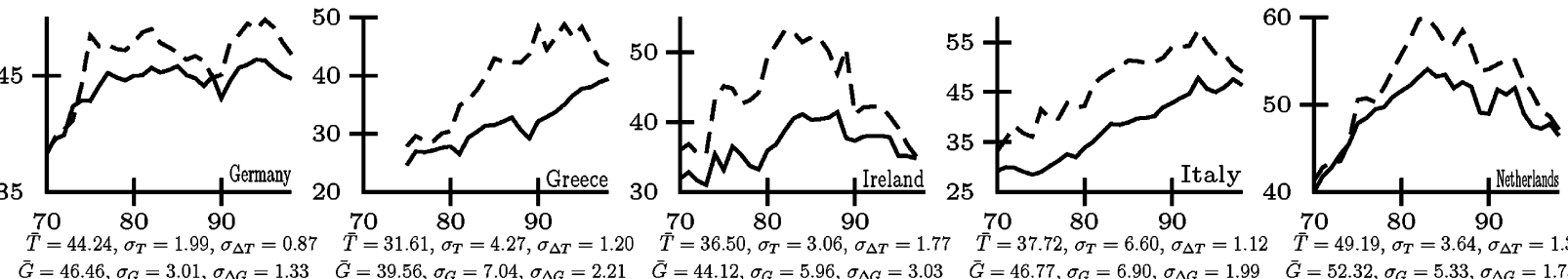

$\bar{G}=46.46, \sigma_{G}=3.01, \sigma_{\Delta G}=1.33 \quad \bar{G}=39.56, \sigma_{G}=7.04, \sigma_{\Delta G}=2.21 \quad \bar{G}=44.12, \sigma_{G}=5.96, \sigma_{\Delta G}=3.03 \quad \bar{G}=46.77, \sigma_{G}=6.90, \sigma_{\Delta G}=1.99 \quad \bar{G}=52.32, \sigma_{G}=5.33, \sigma_{\Delta G}=1.73$
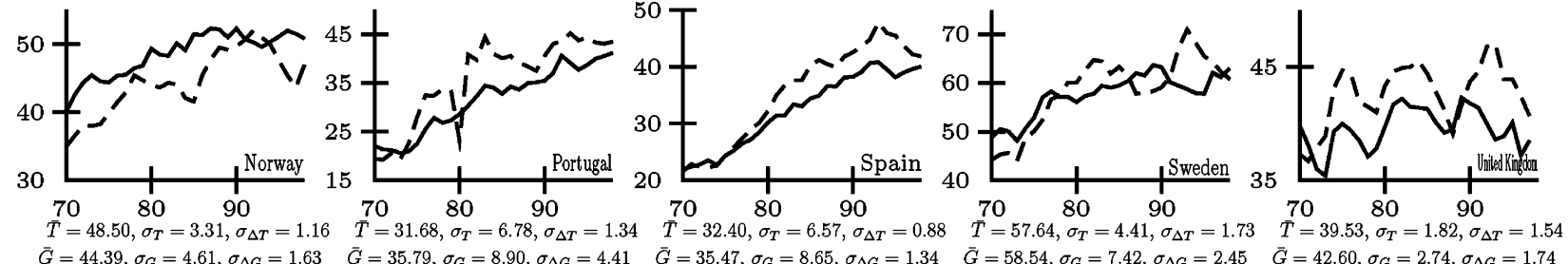

$\bar{G}=44.39, \sigma_{G}=4.61, \sigma_{\Delta G}=1.63 \quad \bar{G}=35.79, \sigma_{G}=8.90, \sigma_{\Delta G}=4.41 \quad \bar{G}=35.47, \sigma_{G}=8.65, \sigma_{\Delta G}=1.34 \quad \bar{G}=58.54, \sigma_{G}=7.42, \sigma_{\Delta G}=2.45 \quad \bar{G}=42.60, \sigma_{G}=2.74, \sigma_{\Delta G}=1.74$

Fig. 2. Stimulus data $(T(-), G(--))$.

fiscal time series. For all countries, expectations started after the seventh year of the stimulus (which was 1977 for most countries). A graph of the stimulus data for the different countries is shown in Fig. 2, with the basic statistics of the two series (means, $\bar{G}$ and $\bar{T}$; standard deviations, $\sigma_{T}$ and $\sigma_{G}$; and standard deviations of the rates of change, $\sigma_{\Delta T}$ and $\sigma_{\Delta G}$ ).

In total, 170 subjects participated in the experiment. Each subject could participate only in one treatment: 76 took part in the baseline $T_{t}$ and $G_{t}$ treatment, 69 in the neutral treatment, and 25 in the $T_{t}$ only treatment.

Each participant made forecasts for a sequence of countries. Sequences were determined randomly and independently for all participants. Table 1 summarises the number of participants playing the various countries in the three treatments.

The experiment was run at the experimental laboratory of the SFB 504 in Mannheim. Participants were students at the university: 116 were from economics or business (we will call them economists), 38 from political science, law and languages (in the following we will call these students in other humanities), and 12 from natural sciences. 130 were male and 40 were female. Sessions were conducted individually and lasted for about $2 \mathrm{~h}$. Participants made, on average, 136 forecasts (between 28 and 309) and completed on average one forecast every 39 s.

Table 1

Countries played by participants across treatments.

\begin{tabular}{|c|c|c|c|c|}
\hline \multirow[t]{2}{*}{ Country } & \multirow[t]{2}{*}{ Sample period } & \multicolumn{3}{|c|}{ Number of participants/country } \\
\hline & & $T_{t}$ and $G_{t}$ & $T_{t}$ only & Neutral \\
\hline Austria & $1970-1998$ & 34 & 14 & 28 \\
\hline Belgium & $1970-1998$ & 52 & 20 & 48 \\
\hline Denmark & 1971-1995 & 43 & 14 & 43 \\
\hline Finland & 1970-1998 & 33 & 12 & 24 \\
\hline France & 1977-1998 & 29 & 10 & 31 \\
\hline Germany & $1970-1998$ & 14 & 13 & 5 \\
\hline Greece & 1975-1998 & 26 & 13 & 22 \\
\hline Ireland & 1970-1995 & 45 & 21 & 44 \\
\hline Italy & $1970-1998$ & 35 & 16 & 29 \\
\hline Netherlands & $1970-1998$ & 32 & 9 & 23 \\
\hline Norway & 1970-1998 & 32 & 14 & 26 \\
\hline Portugal & $1970-1998$ & 25 & 13 & 23 \\
\hline Spain & $1970-1998$ & 30 & 11 & 24 \\
\hline Sweden & 1970-1998 & 53 & 16 & 41 \\
\hline United Kingdom & 1970-1995 & 27 & 15 & 19 \\
\hline Total & & 510 & 211 & 430 \\
\hline
\end{tabular}

Since participants made forecasts for more than one country, the total number is not the sum of the number of participants in all countries. 

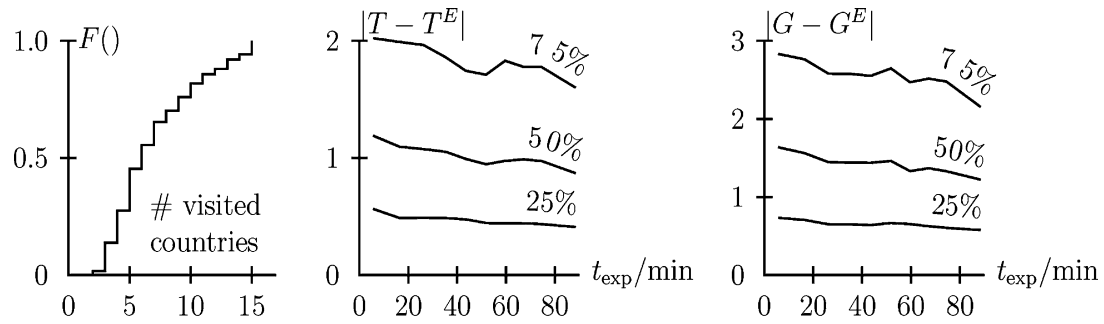

The left graph shows the cumulative frequency of the number of countries our participants visit during the experiment; the middle and the right graph show quantiles for forecast errors during the experiment. Quantiles are taken over ten bands with the same number of observations. The horizontal axis show the total time in minutes since the start of the experiment.

Fig. 3. Countries, learning and fatigue during the experiment. The left graph shows the cumulative frequency of the number of countries our participants visit during the experiment; the middle and the right graph show quantiles for forecast errors during the experiment. Quantiles are taken over 10 bands with the same number of observations. The horizontal axis show the total time in minutes since the start of the experiment.

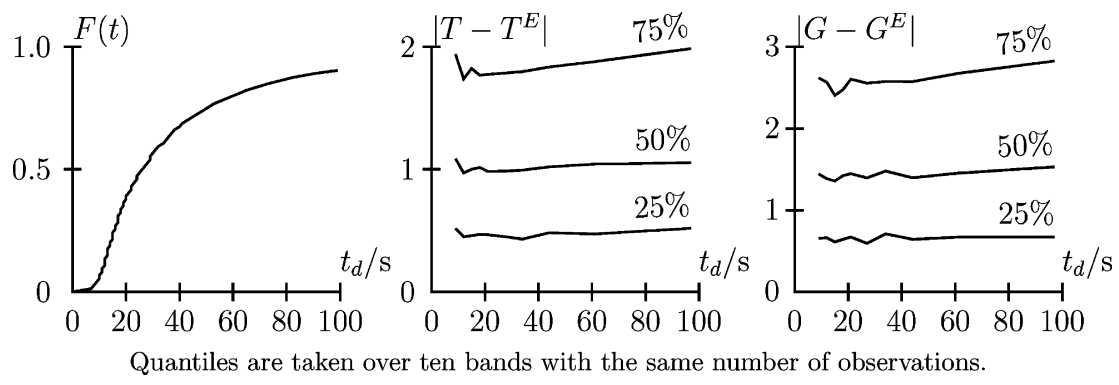

Fig. 4. Distribution of decision time in seconds and quantiles of forecast errors over the decision time. Quantiles are taken over 10 bands with the same number of observations.

\section{Descriptive analysis of the evidence and internal validity}

Laboratory experiments are often inspired by the real world and often use in their setup parameters that are based on field data. Using not only parameters, but datasets from the field in the laboratory is novel and, we believe, innovative, but poses various questions. A first one is about the internal validity of the experimental setup.

\subsection{Learning and fatigue}

The longer an experiment lasts, the more time participants have to learn and to understand their task. However, during a long experiment participants might have difficulties in concentrating; thus the quality of observations might eventually decrease. Fig. 3 shows the development of absolute forecast errors over time. We see that forecast errors decrease over the course of the experiment. The effect of learning seems to dominate fatigue all the time. Though the change over time is rather small, the decreasing trend is significant. ${ }^{6}$

\subsection{Decision time}

The left graph in Fig. 4 shows the cumulative frequency of time subjects take for individual decisions. The mean decision time (over all decisions) is about $35 \mathrm{~s}$ for each forecast; the median (over all participants) is $25 \mathrm{~s}$. While risk loving participants might have a preference to use the maximal forecast time of $2 \mathrm{~min}$, our participants seem to prefer to share their risk over different forecasts. The two remaining graphs in Fig. 4 shows the relation between forecast errors and decision time. The relationship is, if at all, weak. ${ }^{7}$ Harder decisions (characterised by their larger forecast errors) seem to take only slightly more time.

\footnotetext{
${ }^{6}$ Comparing average errors (per participant) in the first and second half of the experiment with a pairwise Wilcoxon test rejects no or an increasing trend with a $p$-value of 0.0003 for $T$ and 0.0015 for $G$. A linear robust model that allows for correlated errors within the same participant rejects with a $p$-value $<10^{-7}$.

7 Separating decision times at the median and comparing average errors as in footnote 6 shows only an insignificant change. A linear robust model yields a $p$-value of 0.098 for $T$ and 0.077 for $G$.
} 

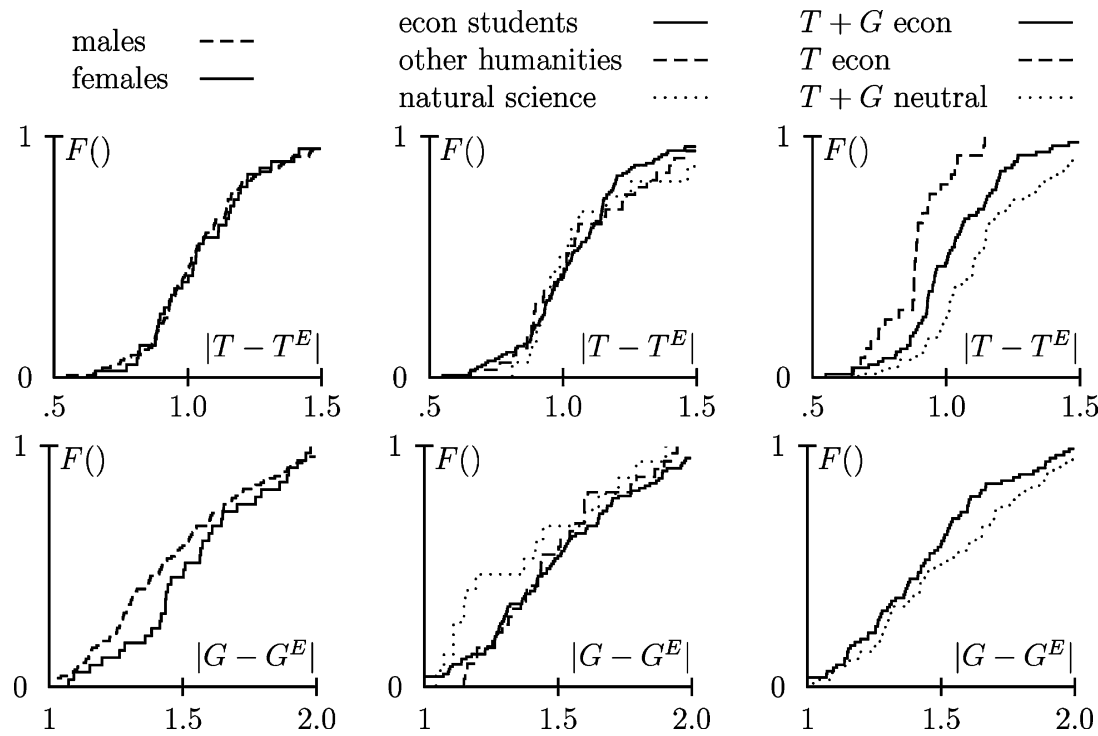

Fig. 5. Distribution of average individual forecast errors by characteristics of participants and by treatment.

\subsection{Participants' characteristics}

The left part of Fig. 5 shows the cumulative distribution of median absolute forecasts errors $\left|T-T^{E}\right|$ and $\left|G-G^{E}\right|$ per participant for male and female participants. The difference is small and not significant. ${ }^{8}$ The centre part of Fig. 5 shows the cumulative distribution of median absolute forecasts errors per participant for different fields. Students from natural sciences seem to make slightly better forecasts for $G$ than the other students. However, these are only 12 out of 170 participants, and the difference between the different fields is small and not significant both for $G$ and for $T .{ }^{9}$

\subsection{Economic context}

To check whether participants understand the fiscal frame, we compare the $T_{t}$ and $G_{t}$ treatment with the neutral treatment. If participants understand and make use of the economic context, we should observe smaller forecast errors in the $T_{t}$ and $G_{t}$ treatment than in the neutral treatment. The right diagram in Fig. 5 shows cumulative distributions of forecast errors for these treatments. We see that, as expected, forecast errors are larger in the neutral treatment; adding economic context contributes significantly to the understanding of the experiment. ${ }^{10}$ We also see that a simpler task that requires forecasts only for $T$ makes forecasts more precise for this variable.

\subsection{Summary regression}

Figs. 3-5 should give a rough impression of the determinants of sizes of forecast errors. To control for characteristics of participants better and the random allocation of countries to participants, Table 2 presents estimates from linear regressions that explain the squared forecast error as a function of various dummies. Model 1 includes a dummy for the type of treatment (neutral/economic context) and a dummy for the forecasted variables ( $T$-only/ $T$ and $G$ ). Model 2 adds a dummy for gender (female is the reference group) and two dummies for the subject of studies (economics is the reference group). Model 3 , finally, adds dummies for each country.

Only two effects are significant. Both were already visible in Fig. 5. First, reducing the number of variables to forecast in the $T_{t}$ only treatment also reduces the error. Second, withholding information about the economic context increases the error. The latter shows that participants understand the economic context of our experiment, at least when we present variables with their economic meaning.

\footnotetext{
${ }^{8}$ Comparing median absolute errors with a Wilcoxon rank sum test yields a $p$-value of 0.8024 for $T$ and of 0.1235 for $G$. A $t$-test yields a $p$-value of 0.8583 for $T$ and of 0.2841 for $G$. Using mean squared errors instead of medians does not change the result considerably.

${ }^{9}$ Comparing median absolute errors with an $F$-test yields a $p$-value of 0.7549 for $T$ and 0.3243 for $G$; a non-parametric Kruskal-Wallis test yields a $p$-value of 0.997 for $T$ and 0.3110 for $G$.

10 Comparing median absolute errors with a non-parametric Wilcoxon rank sum test yields a $p$-value of 0.0000075 for $T$ and 0.09715 for $G$. A $t$-test yields a $p$-value of 0.00001 for $T$ and 0.0488 for $G$.
} 
Table 2

Estimation of squared forecast errors.

\begin{tabular}{|c|c|c|c|c|c|c|}
\hline & \multicolumn{3}{|l|}{$\left(T-T^{E}\right)^{2}$} & \multicolumn{3}{|l|}{$\left(G-G^{E}\right)^{2}$} \\
\hline & Model $1 \hat{\beta} /(\hat{\sigma})$ & Model $2 \hat{\beta} /(\hat{\sigma})$ & Model $3 \hat{\beta} /(\hat{\sigma})$ & Model $1 \hat{\beta} /(\hat{\sigma})$ & Model $2 \hat{\beta} /(\hat{\sigma})$ & Model $3 \hat{\beta} /(\hat{\sigma})$ \\
\hline Neutral & $0.560^{*}(0.219)$ & $0.604^{* *}(0.214)$ & $0.561^{* *}(0.207)$ & $1.071^{*}(0.472)$ & $1.360^{* *}(0.469)$ & $1.054^{* *}(0.346)$ \\
\hline$T_{t}$ only treatment & $-0.651^{* * *}(0.134)$ & $-0.608^{* * *}(0.138)$ & $-0.498^{* * *}(0.121)$ & & & \\
\hline Male & & $-0.080(0.169)$ & $-0.055(0.156)$ & & $-0.950(0.543)$ & $-0.377(0.388)$ \\
\hline Other humanities & & $-0.175(0.181)$ & $-0.094(0.166)$ & & $-0.251(0.627)$ & $0.129(0.453)$ \\
\hline Natural sciences & & $0.382(0.480)$ & $0.502(0.458)$ & & $-1.106(0.693)$ & $0.028(0.591)$ \\
\hline Country dummies & & & Not shown & & & Not shown \\
\hline Constant & $2.777^{* * *}(0.106)$ & $2.906^{* * *}(0.325)$ & $1.117^{* * *}(0.299)$ & $6.369^{* * *}(0.311)$ & $8.005^{* * *}(1.053)$ & $2.151^{*}(0.827)$ \\
\hline
\end{tabular}

Standard deviations are shown in parentheses and are calculated using a robust regression which takes into account correlations of observations for each participant. Similar results can be obtained also for median absolute errors.

* Denote significance at $5 \%$ level.

** Denote significance at $1 \%$ level.

*** Denote significance at $0.1 \%$ level.

\subsection{External significance}

Another important question about the experimental setup concerns its external significance. Consumers in the field form expectations on fiscal policy not only on the basis of spending and taxes, but also through the composition of the government, the date of the next election, economic development, foreign policy, and natural disasters, to name only a few. In the experimental laboratory we are able to neglect all these factors and study $T$ and $G$ in isolation. Any systematic change of expectations that we observe in the lab must be due to a change in $T$ or $G$.

The choice of factors that we study here, $T$ and $G$, is standard in the empirical literature which studies fiscal conduct of countries. Below we refer to an important stream of literature that, since at least the mid-1980s, has used cointegration techniques and the econometrics of Granger causality to study the dynamics of the public budget intertemporal constraint. ${ }^{11}$ In the next section we develop an empirical model of stimuli and of expectation data that coherently ties the present experiment to such econometric practice. Through it we discuss the meaning and implication of general expectation schemes (rational, adaptive, augmented-adaptive), we consider and estimate properties of field data, and we check how these features are perceived by subjects in the experiment.

\section{An econometric analysis of the field-lab data: expectations}

In this section we want to study the model of expectation formation used by participants and the perceived relationships among fiscal variables. We emphasize again that, unlike previous experiments of expectational models that are based on an artificial data generating process, we are using here data from the field. Using an artificial data generating process has the advantage of providing a clear benchmark solution. A disadvantage is that it might be difficult to explain such a process to participants in an experiment. Another problem is that one observes forecasts for abstract time series when one wants to describe forecasting behaviour for real time series. Using data from the field may make it more likely that the forecasting rules participants use in the experiment are close to the rules they would use otherwise with field data. Using data from the field also means that we lose the obvious benchmark solution. With time series that are based on a known abstract model, it would be clear what rational expectations have to be, but what are rational expectations when we use real world data? We now no longer know the benchmark solution; instead we have to estimate one. We acknowledge that this is far from perfect since econometric techniques used to ascertain properties of real data are often less than perfect. Our approach is based on standard techniques used since at least the mid-1980s by economists and econometricians to study the dynamics of fiscal policies. We assume that the data generation processes for both the field and the lab can be taken to be a Vector Autoregressive Process (VAR). We first review the approach and adapt it to this experiment ${ }^{12}$; then we present the evidence based on its estimation.

\subsection{The VAR model of the field-lab data}

\subsubsection{The VAR for the field data (stimuli)}

The field model concerns the stimuli data, that is the vector $x_{t}:=\left(T_{t}, G_{t}\right)^{\prime}$, treated for each country separately. A standard VAR representation of the field systems is given by

$$
\Delta x_{t}=\mu_{x}+\alpha_{x} \beta_{1 x}^{\prime} x_{t-1}+\sum_{\ell=1}^{k-1} \Gamma_{\ell, x x} \Delta x_{t-\ell}+\varepsilon_{x t} .
$$

\footnotetext{
11 See Section 4.1 below for various references to the literature.

12 See for example Johansen (1995) for a modern treatment of the literature on VAR. See our technical report (Bernasconi et al., 2004) for a more detailed exposition of how the field and lab VARs can be consistently derived in the present experiment.
} 
The expression takes the stimuli data $x_{t}:=\left(T_{t}, G_{t}\right)^{\prime}$ as integrated of order $1, \mathrm{I}(1) ; \mu_{x}$ is a vector of constants; $\beta_{1 x}^{\prime} x_{t-1}$ is an error correction term (cointegrating relation $\mathrm{CI}$ ) expressing the long-run disequilibrium between taxes and expenditure in the field; $\alpha_{x}=\left(\alpha_{T}, \alpha_{G}\right)^{\prime}$ is the associated vector of the adjustment coefficients; $\Gamma_{\ell, x x}$ are the matrices of the coefficients of partial adjustments; and $\varepsilon_{x t}$ are error terms i.i.d. $N\left(0, \Omega_{x x}\right)$.

Versions of this basic model have been analysed extensively in the literature (see, e.g. Trehan and Walsh, 1991; Ahmed and Rogers, 1995). An issue considered is sustainability of fiscal policy which can be checked by way of cointegration analysis between taxes and public expenditure. In short, cointegration tests are based on the idea that solvency requires the budget deficit to be stationary. Even when $T_{t}$ and $G_{t}$ are non-stationary, there may be linear combinations of the form $T_{t}+\gamma G_{t}+\rho$ (where $\gamma$ and $\rho$ are constants) which are stationary. In this case, $T_{t}$ and $G_{t}$ are said to be cointegrated of order 1 , with cointegrating vector $(\mathrm{CI})(1, \gamma)$. Stationarity of the budget then further requires $\gamma=-1$ (homogeneity condition).

A second empirical issue analysed within Eq. (3) concerns the direction of causality between taxes and public expenditure. ${ }^{13}$ In particular, when the series in $x_{t}:=\left(T_{t}, G_{t}\right)^{\prime}$ are cointegrated, at least one of $T_{t}$ and $G_{t}$ adjusts to disequilibrium with respect to the long-run relation. Four cases may then emerge: that taxes cause spending, that spending causes taxes, bidirectional causality, and lack of causality. These cases can be represented as a vector $\alpha_{x}=\left(\alpha_{T}, \alpha_{G}\right)^{\prime}$ of the forms $(0$, $\left.{ }^{*}\right)^{\prime},\left({ }^{*}, 0\right)^{\prime},\left({ }^{*},{ }^{*}\right)^{\prime}$ and $(0,0)^{\prime}$ (with * indicating a non-zero coefficient), thus providing tests of Granger long-run causality. ${ }^{14}$ Short-run causality can be similarly checked by looking for significant off-diagonal coefficients in the matrices $\Gamma_{\ell, x x}$.

\subsubsection{The VAR for the lab data (expectations)}

For the lab model, let $m$ indicate the number of individuals, $i=1, \ldots, m$, in a treatment. Recall that expectations $y_{i, t}=$ $\left(T_{t}^{E_{i}}, G_{t}^{E_{i}}\right)^{\prime}$ in the $T_{t}$ and $G_{t}$ treatment and $y_{i, t}=T_{t}^{E_{i}}$ in the $T_{t}$ only treatment. We now illustrate the model for $y_{i, t}=\left(T_{t}^{E_{i}}, G_{t}^{E_{i}}\right)^{\prime}$, which is applied with the obvious modifications to the $T_{t}$ only treatment.

For individual $i$ the VAR has the following form:

$$
\left(\begin{array}{c}
\Delta T_{t}^{E_{i}} \\
\Delta G_{t}^{E_{i}}
\end{array}\right)=\mu_{i}+\alpha_{i}\left(\begin{array}{c}
T_{t-1}+\hat{\gamma} G_{t-1} \\
T_{t-1}^{E_{i}}-T_{t-1} \\
G_{t-1}^{E_{i}}-G_{t-1}
\end{array}\right)+\sum_{\ell=1}^{k-1}\left(\Gamma_{\ell, i i}, \Gamma_{\ell, i x}\right)\left(\begin{array}{c}
\Delta y_{i, t-\ell} \\
\Delta x_{t-\ell}
\end{array}\right)+\hat{\varepsilon}_{y t} .
$$

Eq. (4) includes a vector of constant terms $\mu_{i}=\left(\mu_{i T^{E}}, \mu_{i G^{E}}\right)$; a matrix $(2 \times 3)$ of adjustment coefficients $\alpha_{i}$, decomposed as $\alpha_{i}:=\left(\alpha_{i 1}, \alpha_{i 2}\right)$, where $\alpha_{i 1}=\left(\alpha_{i T^{E}}, \alpha_{i G^{E}}\right)$, shows how the expectation variables may react to the disequilibrium vector estimated in the field, $T_{t-1}+\hat{\gamma} G_{t-1}$ (also labeled ECM ${ }^{T G}$ ); while vec $\left(\alpha_{i 2}^{\prime}\right)^{\prime}=\left(\alpha_{i T^{E}\left(T_{T}{ }_{T}\right)}, \alpha_{i T^{E}\left(G^{E} G\right)}, \alpha_{i G^{E}\left(T^{E} T\right)}, \alpha_{i G^{E}\left(G^{E} G\right)}\right)$ includes the responses to two further possible $\mathrm{CI}$ relations given by the errors in expectations $\left(T_{t-1}^{E_{i}}-T_{t-1}, G_{t-1}^{E_{i}}-G_{t-1}\right)^{\prime}$ (also called (ECM $T^{E} T$, ECM $\left.^{E} G\right)^{\prime}$ ); $\Gamma_{\ell, i x}$ captures causality from the field to the lab, and $\Gamma_{\ell, i i}$ causality from past expectations to present ones.

We should note that for participants who perceive the fiscal properties from the field the lab system (4) collapses to the specification of the marginal field system (3). We use the field system (3) as one reference point and say that participants whose expectations follow (3) have rational expectations. We will also investigate whether and how participants pick up the actual cointegration by comparing $\alpha_{i 1}=\left(\alpha_{i T^{E}}, \alpha_{i G^{E}}\right)$ ' from the lab with $\alpha_{x}=\left(\alpha_{T}, \alpha_{G}\right)^{\prime}$ from the field. Also of interest is the inference on the vector of constant terms $\mu_{i}$, indicating subjects' perception of trends in the data.

One alternative class of expectation formation processes is the adaptive scheme, as originated in the 1950s by the works of different scholars (see Pesaran, 1987 for references). We emphasize the bivariate nature that can take here the adaptive schemes. In particular, under univariate or purely adaptive expectations, past values of the forecasted variable $x_{t}^{1}$ and of its forecast $y_{i, t}^{1}$ enter the expectation process for $y_{i, t}^{1}$, where a superscript 1 indicates one stimulus variable and the corresponding forecast. If other variables enter in the estimated equation for $\Delta y_{i, t}^{1}$, this is evidence against a purely adaptive scheme and in favour of a more general class of models known as augmented-adaptive (see again Pesaran, 1987 for the many variations nested within this definition). ${ }^{15}$ In augmented-adaptive schemes one can also address questions of perceived causality between taxes and public expenditure. ${ }^{16}$

The individual lab sub-systems (4) may be estimated one at the time or jointly. Joint estimation under some homogeneity restrictions allows us to exploit the panel dimension of the data to increase efficiency. In the empirical analysis we will first estimate for each country a model of a representative-agent's expectations. This model we will compare with the corresponding country field model. In this comparison we will focus on the expectations obtained from the experiment conducted under the fiscal frame. Then we will compare results from different treatments, including experiments conducted

\footnotetext{
13 See Garcia and Henin (1999) and Payne (1998) for reviews and recent empirical evidence on this classical theme of public finance.

14 Lack of causality (i.e. $\left.\alpha_{X}=(0,0)^{\prime}\right)$ is incompatible with the presence of cointegration in (3) (see Johansen, 1995).

15 In most previous expectation experiments, subjects had to forecast univariate time series (as for example in Schmalensee, 1976 or Hey, 1994) or reducedunivariate schemes arising from augmented-adaptive forms (as in experiments of price expectations in classical cobweb economies, for example Hommes et al., 2007 or on inflation expectations in overlapping generation economies; see Duffy, 1998 for a review). They generally found support for adaptive expectations (though not necessarily of the first order, see, e.g. Hey).

${ }^{16}$ In particular, by inspection of the parameters in (4) one should note the following: the $\alpha_{i 1}$ coefficients and the off-diagonal elements in the $\Gamma_{\ell}$, ix matrices determine the direction of causality from the field to the lab, while the $\alpha_{i 2}$ coefficients and the off-diagonal elements in the $\Gamma_{\ell, i i}$ matrices regulate the ones from the past expectations on present expectations.
} 
Table 3

Cointegrating vectors estimated for stimulus data.

\begin{tabular}{llc}
\hline Country & Cointegrating vector $\left(\beta_{1 x}^{\prime}, \rho\right)$ & Test of $\hat{\gamma}=-1$ homogeneity condition \\
\hline Austria & $(1,-0.739,-10.385)$ & $-13.23^{* * *}$ \\
Finland & $(1,-0.565,-21.436)$ & $-28.29^{* * *}$ \\
Germany & $(1,-0.572,-17.863)$ & $-4.32^{* *}$ \\
Italy & $(1,-0.892,0)$ & -1.72 \\
Netherlands & $(1,-0.606,-17.630)$ & $-13.51^{* * *}$ \\
Norway & $(1,-1.051,0)$ & $-3.52^{* *}$ \\
Portugal & $(1,-1.177,13.667)$ & $-2.09^{*}$ \\
Sweden & $(1,-0.950,0)$ & $-2.13^{*}$ \\
UK & $(1,-0.922,0)$ & $-9.18^{* * *}$ \\
\hline
\end{tabular}

Belgium, Denmark, France, Greece, Ireland, and Spain are not shown in the table since they show no cointegration.

* Denote $10 \%$ significance level.

** Denote 5\% significance level.

**** Denote $1 \%$ significance level.

under the neutral frame. We will also reconsider the homogeneity condition and we will present estimations at the individual level.

\subsection{Evidence}

\subsubsection{Comparison between the field and the lab VARs}

Table 3 reports the results of the cointegration analysis on the stimulus data. ${ }^{17}$ For 9 countries (Austria, Finland, Germany, Italy, Netherlands, Norway, Portugal, Sweden, and UK), we find that taxes and public expenditures are cointegrated; for 6 (Belgium, Denmark, France, Greece, Ireland, and Spain) we find that they are not. Among the former and consistently with the evidence reported in the literature (see, e.g. Manasse, 1996), the condition for stationarity of the budget $\hat{\gamma}=-1$ is rejected for most countries. The estimated CI vectors have the form $\left(\beta_{1 x}^{\prime}, \rho\right)$ with $\beta_{1 x}^{\prime} x_{t}=T_{t}+\gamma G_{t}$ and $\mu x=\alpha_{x} \cdot \rho$, which exclude linear trends in the data (see Johansen, 1995). This feature of field data is intuitive ${ }^{18}$ and indeed passed for all countries in the sample. Below we will check how subjects reflect this feature of the real data in their expectations.

Table 4 summarises the main findings of the inference on both the field and the lab systems in the two experimental treatments under the fiscal frame. All parameters reported in the table are significant at least at a $5 \%$ level. ${ }^{19}$ In considering the results, recall that in the $T_{t}$ only treatment, in which agents forecast taxes, expectations on $G_{t}^{E_{i}}$ are not available.

The leftmost part of the table reports, for the field and for the lab, the coefficients of responses to the field error correction term ${ }_{\mathrm{ECM}} T G=T_{t}+\hat{\gamma} G_{t}$. Consider first the field evidence (the first column in the table). Among the nine countries for which cointegration between taxes and expenditure was found, expenditure is long-run adjusting to taxes in four cases (Austria, Norway, Sweden and UK); taxes are adjusting to expenditure in four others (Finland, Germany, Italy and Netherlands); and in one country (Portugal) adjustment is bidirectional.

Results from the experiments show that subjects do not follow the CI characteristics of the field data. In both experimental treatments the lab responses $\alpha_{i 1}$ to ${ }_{\mathrm{ECM}} T G$ are often not significantly different from zero, and even when they are, $\alpha_{i 1}$ are in any case quite small. On the contrary, the vectors of constants estimated in the lab show that, while subjects do not follow the $\mathrm{CI}$ characteristics of the real-data, they perceive for most countries a linear trend which is not found by the field VARs.

In the rightmost part of Table 4 we show the results of tests on Granger causality in the field and compare them with tests of perceived causality in the lab. Results speak strongly against subjects correctly perceiving the properties of field stimuli data. In the $T_{t}$ and $G_{t}$ treatment only in one case (Portugal) the causality matches the causality from the field data; in the $T_{t}$ only treatment subjects perceive a good deal of causality from expenditure to taxes, but in many cases it is not consistent with the causality in the field.

\subsubsection{Expectation schemes}

The comparisons between the field and the lab VARs clearly reject the hypothesis of subjects following rational expectations. Looking at the middle part of Table 4 we find instead support for adaptive expectations. Estimates of the vector $\alpha_{i 2}$ describe how individuals react in the long-run to errors in their expectations $\left(T_{t-1}^{E_{i}}-T_{t-1}, G_{t-1}^{E_{i}}-G_{t-1}\right)^{\prime}$. In the $T_{t}$ and $G_{t}$ treatment the coefficients for ${ }_{\mathrm{ECM}} T^{E} T$ in the equation for $\Delta T_{t}^{E_{i}}$ and the coefficients on ${ }_{\mathrm{ECM}} G^{E} G$ in the equation for $\Delta G_{t}^{E_{i}}$ are

\footnotetext{
17 We follow a standard procedure to test for cointegration in system (3). We start determining the lags order $k$ of the field VAR (which was two for most countries) and then use the Johansen procedure to test for the rank $r$ of the system (that is, the number of $\mathrm{CI}$ vectors). We use the LR trace test for $H_{0}: r=0$ versus $H_{1}: r=1$ and exclude the case of a stationary system $r=2$, where both taxes and expenditure are stationary in levels.

18 Since $x_{t}$ contains the ratio of taxes and expenditure to total GDP, we expect the system $x_{t}:=\left(T_{t}, G_{t}\right)^{\prime}$ not to contain a linear trend.

19 Detailed parameter estimates, together with standard errors and level of statistical significance, are available in Bernasconi et al. (2004). All models presented here have been selected performing a computerised strategy, which started to drop coefficients with higher $p$-values. We have checked that the selected models are robust to alternative procedures.
} 
Table 4

Summary of inference results.

\begin{tabular}{|c|c|c|c|c|c|c|c|c|c|c|}
\hline & \multicolumn{3}{|c|}{ Vectors of responses to $\mathrm{ECM} T G: T_{t}+\hat{\gamma} G_{t}$} & \multicolumn{2}{|c|}{$\begin{array}{l}\text { Vectors of lab constants } \\
\mu_{i}=\left(\mu_{i T^{E}}: \mu_{i G^{E}}\right)\end{array}$} & \multicolumn{2}{|c|}{$\begin{array}{l}\text { Vectors of responses to } \\
\left(\underline{E C E} T^{E} T,{ }_{\text {ECE }} G^{E} G\right)^{\prime}:\left(T_{t}^{E_{i}}-T_{t}, G_{t}^{E_{i}}-G_{t}\right)^{\prime}\end{array}$} & \multicolumn{3}{|c|}{$\begin{array}{l}\text { Direction of short-run causality } \\
\text { inferred from } \Gamma_{\ell, x x} \text { and } \Gamma_{\ell, i x}\end{array}$} \\
\hline & Field $\alpha_{x}^{\prime}$ & $\begin{array}{l}T_{t} \text { and } G_{t} \\
\text { treatment } \alpha_{i 1}^{\prime}\end{array}$ & $\begin{array}{l}T_{t} \text { only } \\
\text { treatment } \alpha_{i 1}^{\prime} \\
\end{array}$ & $\begin{array}{l}T_{t} \text { and } G_{t} \\
\text { treatment } \mu_{i}^{\prime}\end{array}$ & $\begin{array}{l}T_{t} \text { only } \\
\text { treatment } \mu_{i}^{\prime}\end{array}$ & $T_{t}$ and treatment $\alpha_{i 2}$ & $\begin{array}{l}T_{t} \text { only } \\
\text { treatment } \alpha_{i 2}\end{array}$ & Field & $\begin{array}{l}T_{t} \text { and } G_{t} \\
\text { treatment }\end{array}$ & $\begin{array}{l}T_{t} \text { only } \\
\text { treatment }\end{array}$ \\
\hline Austria & $(0,1.308)$ & $(0,0)$ & $\left(0,{ }^{*}\right)$ & $(0.264,0.146)$ & $\left(0.155,{ }^{*}\right)$ & $((-0.921,0),(0.084,-0.695))$ & $\left(\left(-0.863,{ }^{*}\right),{ }^{*}\right)$ & $T \leftrightarrow G$ & & $T \leftarrow G$ \\
\hline Belgium & & & & $(0.285,0.138)$ & $(0.160, *)$ & $((-0.838,0),(0,-0.656))$ & $\left(\left(-0.810,{ }^{*}\right),{ }^{*}\right)$ & $T \leftarrow G$ & $T \leftrightarrow G$ & $T \leftarrow G$ \\
\hline Denmark & & & & $(0.273,0.172)$ & $\left(0,{ }^{*}\right)$ & $((-0.885,0.138),(0.096,-0.622))$ & $\left(\left(-0.872,{ }^{*}\right),{ }^{*}\right)$ & & $T \leftrightarrow G$ & $T \leftarrow G$ \\
\hline Finland & $(-0.953,0)$ & $(-0.145,0)$ & $\left(-0.164,{ }^{*}\right)$ & $(0,0)$ & $\left(0,{ }^{*}\right)$ & $((-0.923,0.171),(0,-0.512))$ & $\left(\left(-0.825,{ }^{*}\right),{ }^{*}\right)$ & $T \leftarrow G$ & $T \leftrightarrow G$ & $T \leftarrow G$ \\
\hline France & & & & $(0.175,0)$ & $\left(0,{ }^{*}\right)$ & $((-0.709,-0.124)(0,-0.849))$ & $\left(\left(-0.655,{ }^{*}\right),{ }^{*}\right)$ & $T \leftarrow G$ & $T \leftrightarrow G$ & $T \leftarrow G$ \\
\hline Germany & $(-1.007,0)$ & $(0,0)$ & $(0.165, *)$ & $(0.199,0.329)$ & $\left(0.110,{ }^{*}\right)$ & $((-0.842,0),(0,-0.695))$ & $\left(\left(-0.961,{ }^{*}\right),{ }^{*}\right)$ & & $T \rightarrow G$ & $T \leftarrow G$ \\
\hline Greece & & & & $(0.606,0.199)$ & $\left(0.581,{ }^{*}\right)$ & $((-0.915,0),(0,-0.717))$ & $\left(\left(-1.058,{ }^{*}\right), *\right)$ & & $T \leftarrow G$ & $T \leftarrow G$ \\
\hline Ireland & & & & $(0.435,0.561)$ & $\left(0.322,{ }^{*}\right)$ & $((-0.739,0),(-0.141,-0.799))$ & $\left(\left(-0.676,{ }^{*}\right),{ }^{*}\right)$ & & $T \rightarrow G$ & $T \leftarrow G$ \\
\hline Italy & $(-0.155,0)$ & $\begin{array}{l}(-0.072 \\
-0.084)\end{array}$ & $\left(-0.034,{ }^{*}\right)$ & $(0,0)$ & $\left(0,{ }^{*}\right)$ & $((-0.771,0.074),(0,-0.744))$ & $\left(\left(-0.864,{ }^{*}\right),{ }^{*}\right)$ & & $T \rightarrow G$ & \\
\hline Netherlands & $(-0.623,0)$ & $(0,0)$ & $\left(0,{ }^{*}\right)$ & $(0.280,0.301)$ & $\left(0.324,{ }^{*}\right)$ & $((-0.736,0),(0,-0.800))$ & $\left(\left(-0.809,{ }^{*}\right),{ }^{*}\right)$ & & $T \leftarrow G$ & $T \leftarrow G$ \\
\hline Norway & $(0,0.308)$ & $(0,0.083)$ & $\left(0,{ }^{*}\right)$ & $(0,0.314)$ & $\left(0,{ }^{*}\right)$ & $((-0.732,0),(0.166,-0.751))$ & $\left(\left(-0.731,{ }^{*}\right),{ }^{*}\right)$ & $T \rightarrow G$ & $T \rightarrow G$ & $T \leftarrow G$ \\
\hline Portugal & $(0.290,0.670)$ & $(0,0.175)$ & $\left(0,{ }^{*}\right)$ & $(0.711,0)$ & $(0.175, *)$ & $((-0.744,0),(0.240,-0.633))$ & $\left((-0.758, *),{ }^{*}\right)$ & $T \leftrightarrow G$ & $T \leftrightarrow G$ & $T \leftarrow G$ \\
\hline Spain & & & & $(0.276,0.141)$ & $\left(0.202,{ }^{*}\right)$ & $((-0.732,0),(0,-0.657))$ & $\left(\left(-0.548,{ }^{*}\right),{ }^{*}\right)$ & & $T \rightarrow G$ & $T \leftarrow G$ \\
\hline Sweden & $(0,0.280)$ & $\begin{array}{l}(-0.034 \\
0.086)\end{array}$ & $\left(0,{ }^{*}\right)$ & $(0.239,0.138)$ & $\left(0.112,{ }^{*}\right)$ & $((-0.692,0),(0.111,-0.743))$ & $\left(\left(-0.670,{ }^{*}\right),{ }^{*}\right)$ & $T \rightarrow G$ & $T \leftarrow G$ & $T \leftarrow G$ \\
\hline United-Kingdom & $(0,0.435)$ & $(0,0)$ & $\left(0,{ }^{*}\right)$ & $(0.249,0.176)$ & $\left(0.172,{ }^{*}\right)$ & $((-0.883,0),(0.175,-0.780))$ & $\left(\left(-0.675,{ }^{*}\right),{ }^{*}\right)$ & & $T \rightarrow G$ & $T \leftarrow G$ \\
\hline
\end{tabular}




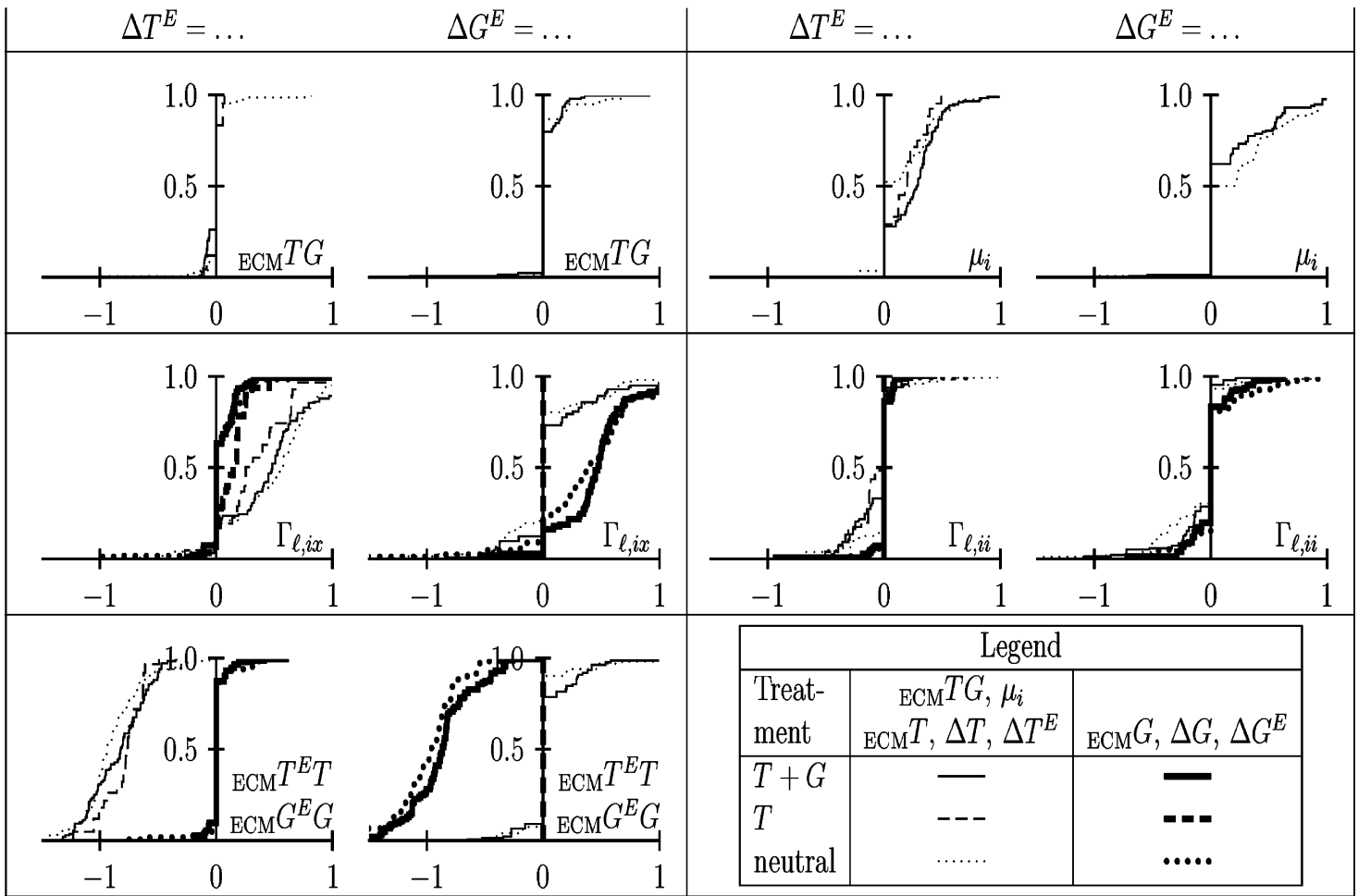

Fig. 6. Results of estimating Eq. (4) for each individual. Graphs show the distribution of the estimated coefficients of Eq. (4). We impose the restriction that each individual uses the same model for all countries.

positive and close to 1 . The same holds in the $T_{t}$ only treatment for the coefficients for ${ }_{\mathrm{ECM}} T^{\mathrm{E}} T$ in the equation for $\Delta T_{t}^{E_{i}}$. In both experimental treatments the estimated parameters are typically less than 1 . (This is also confirmed by formal $t$-tests conducted on the parameters.) Conversely, in the $T_{t}$ and $G_{t}$ treatment, the off-diagonal diagonal of $\alpha_{i 2}$ are in most cases equal to or very close to zero. In other words, subjects do not adjust expectations for taxes to errors in the expectations on expenditure, and vice versa.

To obtain further evidence on the process subjects use to form expectations, we estimate Eq. (4) separately for each individual. ${ }^{20}$ Fig. 6 presents distributions of the coefficients for the two fiscal treatments as well as for the neutral treatment. Results confirm what we learned from the aggregate model. Coefficients of responses to the field error correction term ECM $T G$ are zero or close to zero for all treatments and for both equations. Positive liner trends $\left(\mu_{i}\right)$ in the data are perceived by several participants. The coefficients of matrices $\Gamma_{\ell, i x}$ summed over lags show that, in both equations, the diagonal coefficients (hence the own-effects of $\Delta T_{t-\ell}$ on $\Delta T_{t}^{E_{i}}$ and of $\Delta G_{t-\ell}$ on $\Delta G_{t}^{E_{i}}$ ) are larger than the off-diagonal terms, but the latter do not necessarily vanish. The coefficients for $\Gamma_{\ell, i i}$, summed-up over lags, refer to the short-run effects of past expectations on present expectations and show a moderate tendency of subjects to smooth out peaks in past expectations. The distributions of the adjustment coefficients to the error terms $\left(\mathrm{ECM}^{E} T, \mathrm{ECM}^{E} G^{E}\right)^{\prime}$ confirm that in all treatments subjects adjust to past forecast errors.

As a whole, we take the above results to support the hypothesis of subjects following adaptive expectations, but not quite a purely univariate model. While the results suggest that participants assume some interaction between fiscal variables, we can conclude from Table 4 that this interaction changes over countries. ${ }^{21}$ We take this to be evidence in favor of models of augmented-adaptive expectations.

In Section 3 we have compared different treatment frames. In our baseline frame the variables shown on the screen have their original economic labels. In the alternative 'neutral' frame these labels are just letters from A to D. We suspected that forecasts in the 'neutral' frame are less precise and, indeed, confirmed this hypothesis in Fig. 5 and in Table 2. In the same section we compared different participant groups. Several of our participants are students in economics or business administration. We found, however, that forecasts of these participants are not better than those of other participants.

Can one use the estimates of Eq. (4) in order to learn more about why economic labels help participants to make forecasts? If participants from economics are not systematically better in their forecasts, are they at least systematically different? Unfortunately, the answer to both questions is No.

\footnotetext{
20 To select the model we use the same procedure as in the aggregate model (footnote 19).

21 This can be confirmed be estimating Eq. (4) separately for individuals and countries.
} 
Table 5

Impact of treatment frame and participants on Eq. (4).

\begin{tabular}{|c|c|c|c|c|c|c|c|c|c|c|c|c|}
\hline \multirow{3}{*}{$\begin{array}{l}\text { Data comparison } \\
\text { dep. var. coefficient }\end{array}$} & \multicolumn{6}{|c|}{$T_{t}$ and $G_{t}$ treatment frame: neutral $\leftrightarrow$ economic labels } & \multicolumn{6}{|c|}{$T_{t}$ and $G_{t}$ treatment and economics $\leftrightarrow$ other fields } \\
\hline & \multicolumn{3}{|l|}{$\Delta T^{E}$} & \multicolumn{3}{|l|}{$\Delta G^{E}$} & \multicolumn{3}{|l|}{$\Delta T^{E}$} & \multicolumn{3}{|l|}{$\Delta G^{E}$} \\
\hline & Econ & Neutral & $p$ & Econ & Neutral & $p$ & Other & Econ & $p$ & Other & Econ & $p$ \\
\hline $\mathrm{ECM}^{T G}$ & -0.021 & -0.008 & 0.543 & 0.026 & 0.052 & 0.390 & -0.021 & -0.021 & 1.00 & 0.030 & 0.025 & 0.879 \\
\hline$\mu_{i}$ & 0.268 & 0.199 & 0.109 & 0.170 & 0.271 & 0.136 & 0.274 & 0.266 & 0.888 & 0.198 & 0.159 & 0.642 \\
\hline$\Delta T: \Gamma_{\ell, i x}$ & 0.395 & 0.442 & 0.478 & 0.082 & 0.077 & 0.961 & 0.500 & 0.350 & 0.068 & 0.144 & 0.055 & 0.324 \\
\hline$\Delta G: \Gamma_{\ell, i x}$ & 0.069 & 0.036 & 0.315 & 0.425 & 0.306 & 0.114 & 0.057 & 0.074 & 0.630 & 0.582 & 0.357 & 0.013 \\
\hline$\Delta T: \Gamma_{\ell, i i}$ & -0.049 & -0.002 & 0.149 & -0.063 & -0.091 & 0.561 & -0.076 & -0.037 & 0.375 & -0.095 & -0.050 & 0.335 \\
\hline$\Delta G: \Gamma_{\ell, i i}$ & -0.009 & 0.008 & 0.352 & 0.018 & 0.062 & 0.226 & -0.008 & -0.009 & 0.987 & -0.044 & 0.045 & 0.052 \\
\hline$\Delta T: \mathrm{ECM}^{E .}$ & -0.881 & -0.918 & 0.437 & 0.038 & 0.062 & 0.623 & -0.805 & -0.914 & 0.098 & 0.101 & 0.010 & 0.083 \\
\hline$\Delta G: \mathrm{ECM}^{E .}$ & 0.016 & -0.005 & 0.319 & -0.910 & -1.021 & 0.035 & 0.028 & 0.011 & 0.504 & -0.813 & -0.953 & 0.057 \\
\hline
\end{tabular}

The table reports average estimated coefficients of Eq. (4). The left part compares the neutral and the economic frame. The right part compares the average coefficients for participants with a background in economics with those from other disciplines. Next to the two means (for 'other' and 'econ') the table reports a $p$-value for a Welch two sample $t$-test.

Table 5 compares average coefficients of Eq. (4) for the treatment with neutral and economic labels and for the participants with different backgrounds. Most differences between coefficients are not significant.

\section{Conclusions}

Experimental economics has grown substantially over the last two or three decades, as it is now a well-acknowledged method through which decision theorists, game theorists and microeconomists have tested and refined theoretical models in their respective fields of interest.

Relatively few experiments have been conducted in the field of macroeconomics. The reason, probably, is that macroeconomists, dealing with real world questions to a much greater degree than other economists, are convicted that laboratory experiments cannot really answer such type of questions. The approach pursued in this paper follows the idea that using real world data as stimuli for subjects in the experiments might be a step in understanding which variables and forces are more relevant when individuals take actions of macroeconomic relevance.

We propose our experiment as an alternative to experiments that are based on entirely artificial time series or time series whose parameters are based on field data. In our experiment participants receive as stimuli real world data on fiscal variables and form expectations on the basis of that information. Such an extension has advantages as well as disadvantages. Constructing a rational reference point is harder than with artificial time series. However, we have seen in our experiment that using economically meaningful data is important as it affects expectations of participants in our experiment.

\section{Appendix A. Supplementary data}

Supplementary data associated with this article can be found, in the online version, at doi:10.1016/j.jebo.2008.11.002.

\section{References}

Ahmed, S., Rogers, J.H., 1995. Government budget deficits and trade deficits: are present value constraints satisfied in long-term data? Journal of Monetary Economics 36, 351-374.

Allers, M., de Haan, J., de Kam, F., 1998. Using survey data to test for Ricardian equivalence. Public Finance Review 26, 565-582.

Alm, J., McKee, M., 2004. Tax compliance as a coordination game. Journal of Economic Behavior and Organization 54 (3), $297-312$.

Bernasconi, M., Kirchkamp, O., 2000. Why do monetary policies matter? An experimental study of saving and inflation in an overlapping generations model. Journal of Monetary Economics 46, 315-343.

Bernasconi, M., Kirchkamp, O., Paruolo, P., 2004. Do fiscal variables affect fiscal expectations? Experiments with real world and lab data. Discussion Paper 04-27, University of Mannheim, SFB 504, http://www.kirchkamp.de/pdf/04-27.pdf.

Blinder, A.S., Morgan, J., 2005. Are two heads better than one? Monetary policy by committee. Journal of Money Credit and Banking 37 (5), $789-811$.

Costa-Gomes, M., Crawford, V.P., 2001. Cognition and behavior in normal-form games: an experimental study. Econometrica 69 (5), $1193-1235$.

Croson, R., 2000. Thinking like a game theorist: factors affecting the frequency of equilibrium play. Journal of Economic Behavior and Organization 41 (3), 299-314.

Duffy, J., 1998. Monetary theory in the laboratory. Federal Reserve Bank of St. Louis Review 80, 9-26.

Fisher, F.M., 1962. A Priori Information and Time Series Analysis. North-Holland, Amsterdam.

Garcia, S., Henin, P.-Y., 1999. Balancing budget through tax increases or expenditure cuts: is it neutral? Economic Modelling 16, 591-612.

Grun, D., 1991. What people know and what economists think they know: surveys on Ricardian equivalence. Australian Economic Papers 30, 1-9.

Hey, J.D., 1994. Expectations formations: rational or adaptive or. ... Journal of Economic Behavior and Organization 25, 329-349.

Hommes, C., Sonnemans, J., Tuinistra, J., van de Velden, H., 2007. Learning in Cobweb experiments. Macroeconomic Dynamics 11, 8-33.

Johansen, S., 1995. Likelihood-based inference in cointegrated vector autoregressive models. In: Advanced Texts in Econometrics. Oxford University Press, Oxford and New York.

Lombardelli, C., Talbot, J., Proudman, J., 2002. Committees versus individuals: an experimental analysis of monetary policy decision-making. Bank of England Quarterly Bulletin 42 (3), 262-273.

Manasse, P., 1996. Are taxes too low? Journal of Economic Dynamics and Control 20, 1263-1288.

Marimon, R., Sunder, S., 1993. Indeterminacy of equilibria in a Hyperinflationary World: experimental evidence. Econometrica 61, $1073-1107$. 
OECD, 2000. Fiscal positions and business cycles. In: CD-ROM Statistical Compendium. OECD, Paris.

Offerman, T., Sonnemans, J., Schram, A., 1996. Value orientations, expectations and voluntary contributions in public goods. Economic Journal 106 (437), 817-845.

Payne, J.E., 1998. The tax-spend debate: time series evidence from state budgets. Public Choice 95, 307-320.

Pesaran, M.H., 1987. The Limits to Rational Expectations. Blackwell, Oxford and New York.

Schmalensee, R., 1976. An experimental study of expectation formation. Econometrica 44, 17-41.

Seater, J.J., 1993. Ricardian equivalence. Journal of Economic Literature 31, 142-190.

Swenson, C.W., 1997. Rational expectation and tax policy: experimental market evidence. Journal of Economic Behavior and Organization 32, 433-455.

Trehan, B., Walsh, C.E., 1991. Testing intertemporal budget constraints: theory and applications to U.S. Federal Budget and Current Account Deficits. Journal of Money, Credit, and Banking 23, 206-223. 